\title{
Properties of a class of mean dependent on a parameter
}

\section{Paul Bracken ${ }^{1 *}(10$}

\section{"Correspondence:}

paul.bracken@utrgv.edu

${ }^{1}$ Department of Mathematics,

University of Texas, Edinburg, USA

\begin{abstract}
Some properties of a new class of binary symmetric mean $M_{p}(a, b)$ which depends on two positive numbers $a$ and $b$, as well as a positive parameter $p$, are investigated. The logarithmic mean and arithmetic mean are two members of this class. It is shown that, for all values of the parameter $p$, the set of $p$-dependent means $M_{p}(a, b)$ is bounded above by the mean $M_{\infty}(a, b)$.
\end{abstract}

MSC: $33 \mathrm{E} 20 ; 40 \mathrm{~A} 30$

Keywords: Means; Series; Convergence; Arithmetic; Bounded

\section{Introduction}

The arithmetic-geometric mean which was first investigated by Lagrange and Gauss [1] has found many applications due to its rapid convergence properties. It has played an important role in the calculation of the number $\pi$, as well as various elliptic integrals.

Let $a$ and $b$ denote real numbers such that $a>b>0$. A sequence of arithmetic means and a sequence of geometric means can be constructed by letting $a_{0}=a$ and $b_{0}=b$ and defining the recursions [2]

$$
a_{n+1}=\frac{1}{2}\left(a_{n}+b_{n}\right), \quad b_{n+1}=\sqrt{a_{n} b_{n}}, \quad n=0,1,2, \ldots
$$

The sequence $\left(b_{n}\right)$ is increasing and bounded above by $a$ while the sequence $\left(a_{n}\right)$ is decreasing and bounded below by $b$ since inductively

$$
b<\cdots<b_{n}<a_{n}<\cdots<a .
$$

Therefore, each sequence converges by the monotone convergence theorem. Since it is the case that [3]

$$
0 \leq a_{n+1}-b_{n+1} \leq \frac{1}{2} \cdot \frac{\left(a_{n}-b_{n}\right)^{2}}{\left(\sqrt{a_{n}}+\sqrt{b_{n}}\right)^{2}},
$$

we observe that $a_{n}$ and $b_{n}$ converge to a common limit determined uniquely by $a_{0}$ and $b_{0}$. The arithmetic-geometric mean $A G(a, b)$ is defined as the common limit of these two

(c) The Author(s) 2019. This article is distributed under the terms of the Creative Commons Attribution 4.0 International License (http://creativecommons.org/licenses/by/4.0/), which permits unrestricted use, distribution, and reproduction in any medium, provided you give appropriate credit to the original author(s) and the source, provide a link to the Creative Commons license, and indicate if changes were made. 
sequences

$$
A G(a, b)=\lim _{n \rightarrow \infty} a_{n}=\lim _{n \rightarrow \infty} b_{n}
$$

Gauss was able to prove that $A G(a, b)$ can be calculated by means of an integral

$$
\frac{1}{A G(a, b)}=\frac{2}{\pi} \int_{0}^{\infty} \frac{d x}{\sqrt{\left(x^{2}+a^{2}\right)\left(x^{2}+b^{2}\right)}}
$$

Another type of mean which also has many applications is the logarithmic mean $L(a, b)$ defined to be

$$
\frac{1}{L(a, b)}=\frac{\log (a)-\log (b)}{a-b}=\int_{0}^{\infty} \frac{d x}{(x+a)(x+b)} .
$$

The similarity of expressions (3) and (4) motivates the introduction of a parametrized set of means which depends on a positive parameter and incorporates these two means. Let $a, b>0$ be given real numbers and $p \in(0, \infty)$ then define $M_{p}(a, b)$ by the integral [4]

$$
\frac{1}{M_{p}(a, b)}=c_{p} \int_{0}^{\infty} \frac{d x}{\left.\left[\left(x^{p}+a^{p}\right)\left(x^{p}+b^{p}\right)\right)\right]^{1 / p}}, \quad 0<p<\infty .
$$

The arithmetic-geometric mean is obtained from (5) by taking $p=2$ and the logarithmic mean is the case $p=1$. The constant $c_{p}$ depends only on the single parameter $p$ and is defined to satisfy the condition $M_{p}(a, a)=a$. Expressed as an integral it takes the form

$$
\frac{1}{c_{p}}=a \int_{0}^{\infty} \frac{d x}{\left(x^{p}+a^{p}\right)^{2 / p}}=\int_{0}^{\infty} \frac{d t}{\left(t^{p}+1\right)^{2 / p}} .
$$

It is the intention here to study some of the properties of $1 / c_{p}$ and $M_{p}(a, b)[5,6]$. It will be shown that $1 / c_{p}$ increases monotonically for all $p \in(0, \infty)$. Some bounds for the means $M_{p}(a, b)$ are obtained. Two additional means $M_{0}(a, b)$ and $M_{\infty}(a, b)$ are defined by

$$
M_{0}(a, b)=\lim _{p \rightarrow 0^{+}} M_{p}(a, b), \quad M_{\infty}(a, b)=\lim _{p \rightarrow \infty} M_{p}(a, b) .
$$

These two limits will be calculated in closed form. It is shown that $M_{p}(a, b) \leq M_{\infty}(a, b)$ for all $p \in(0, \infty)$.

In general, a binary symmetric mean $M(a, b)$ of positive numbers $a$ and $b$ is a function that satisfies the following properties: (i) $\min (a, b) \leq M(a, b) \leq \max (a, b)$; (ii) $M(a, b)=$ $M(b, a)$; (iii) $M(\lambda a, \lambda b)=\lambda M(a, b)$ for all $\lambda>0$; and (iv) $M(a, b)$ is nondecreasing in $a$ and $b$. It is the case that $M_{p}(a, b)$ satisfies (ii)-(iv), and by the end it will be seen all (i)-(iv) hold [7].

To start let us establish some general bounds for $M_{p}(a, b)$ in an elementary way.

Theorem 1 Let $a, b>0$, then

$$
\min (a, b) \leq \sqrt{a b} \leq M_{p}(a, b) \leq\left(\frac{a^{p}+b^{p}}{2}\right)^{1 / p} \leq \max (a, b)
$$


Proof The first inequality on the left follows from the fact that $\min (a, b) \leq \sqrt{\min (a, b)^{2}} \leq$ $\sqrt{a b}$. The last inequality on the right follows from the fact that $\left(\left(a^{p}+b^{p}\right) / 2\right)^{1 / p}$ is strictly increasing with $p$ and the fact that

$$
\lim _{p \rightarrow \infty}\left(\frac{a^{p}+b^{p}}{2}\right)^{1 / p}=\max (a, b)
$$

Since $\left(a^{p / 2}-b^{p / 2}\right)^{2} \geq 0$, it follows that $2(a b)^{p / 2} \leq a^{p}+b^{p}$. Squaring this, we have further $4(a b)^{p}<\left(a^{p}+b^{p}\right)^{2}$. The following inequalities follow by first adding $x^{2 p}$ and then $a^{p} b^{p}$ to both sides of this result:

$$
\begin{aligned}
x^{2 p}+2(\sqrt{a b})^{p} x^{p}+a^{p} b^{p} & \leq x^{2 p}+\left(a^{p}+b^{p}\right) x^{p}+a^{p} b^{p} \\
& =\left(x^{p}+a^{p}\right)\left(x^{p}+b^{p}\right) \leq x^{2 p}+\left(a^{p}+b^{p}\right) x^{p}+\frac{1}{4}\left(a^{p}+b^{p}\right)^{2} .
\end{aligned}
$$

This is equivalent to the inequalities

$$
\left(x^{p}+(\sqrt{a b})^{p}\right)^{2 / p} \leq\left[\left(x^{p}+a^{p}\right)\left(x^{p}+b^{p}\right)\right]^{1 / p} \leq\left(x^{p}+\frac{a^{p}+b^{p}}{2}\right)^{2 / p} .
$$

Inverting these inequalities and then integrating with respect to $x$, we get

$$
\int_{0}^{\infty} \frac{d x}{\left(x^{p}+\frac{a^{p}+b^{p}}{2}\right)^{2 / p}} \leq \int_{0}^{\infty} \frac{d x}{\left[\left(x^{p}+a^{p}\right)\left(x^{p}+b^{p}\right)\right]^{1 / p}} \leq \int_{0}^{\infty} \frac{d x}{\left(x^{p}+(\sqrt{a b})^{p}\right)^{2 / p}} .
$$

Multiply through (10) by $c_{p}$, invert what results, and then use definition (5) of $M_{p}(a, b)$ to obtain the result

$$
M_{p}(\sqrt{a b}, \sqrt{a b}) \leq M_{p}(a, b) \leq M_{p}\left(\left(\frac{a^{p}+b^{p}}{2}\right)^{1 / p},\left(\frac{a^{p}+b^{p}}{2}\right)^{1 / p}\right) .
$$

Using $M_{p}(z, z)=z$ for any $z>0$, the two inner inequalities in (8) are obtained.

\section{Series representations for the reciprocal of $M_{p}(a, b)$}

Some useful integral and series representations related to these means will be developed next. First, make the substitution $t=\left(y^{p}+1\right)^{-1}$ with $d y=-p^{-1} t^{-1-1 / p}(1-t)^{1 / p-1} d t$ so $c_{p}$ can be put in the form of the beta function integral

$$
\frac{1}{c_{p}}=\frac{1}{p} \int_{0}^{1} t^{1 / p-1}(1-t)^{1 / p-1} d t=\frac{\Gamma\left(\frac{1}{p}\right)^{2}}{p \Gamma\left(\frac{2}{p}\right)} .
$$

Returning to the integral for $M_{p}(a, b)$, let us make the following change of variable:

$$
x^{p}=a^{p}\left(\frac{1}{t}-1\right)=a^{p}\left(\frac{1-t}{t}\right), \quad d x=-\frac{a}{p} t^{-1-1 / p}(1-t)^{1 / p-1} d t .
$$

In this case, the integral for $M_{p}(a, b)$ takes the following form:

$$
\frac{1}{M_{p}(a, b)}=c_{p} \frac{a}{p} \int_{0}^{1} \frac{t^{1-1 / p}(1-t)^{1 / p-1}}{\frac{a}{t^{1 / p}}\left(a^{p} \frac{1-t}{t}+b^{p}\right)^{1 / p}} d t=\frac{c_{p}}{p} \int_{0}^{1} \frac{t^{1 / p-1}(1-t)^{1 / p-1}}{\left(a^{p}(1-t)+b^{p} t\right)^{1 / p}} d t .
$$


Theorem 2 The function $1 / c_{p}$ increases monotonically for all $p \in(0, \infty)$. Moreover, as $p \rightarrow$ $\infty$, this function admits the asymptotic expansion

$$
\frac{1}{c_{p}}=2-\frac{\pi^{2}}{2 p^{2}}+\frac{4 \zeta(3)}{p^{3}}+O\left(\frac{1}{p^{4}}\right)
$$

Proof Beginning with (12) and setting $h(p)=1 / c_{p}$, we have

$$
\log (h(p))=\log \left(\frac{\Gamma\left(\frac{1}{p}\right)^{2}}{p \Gamma\left(\frac{2}{p}\right)}\right)
$$

Differentiating both sides of this with respect to $p$, it follows that

$$
\frac{h^{\prime}(p)}{h(p)}=\frac{1}{p^{2}}\left(2 \psi\left(\frac{2}{p}\right)-2 \psi\left(\frac{1}{p}\right)-p\right) .
$$

It suffices to show the quantity in brackets is always positive. To this end, substitute the series form of $\psi(z)$ to obtain

$$
\begin{aligned}
2 \psi & \left(\frac{2}{p}\right)-2 \psi\left(\frac{1}{p}\right)-p \\
& =-p+\frac{2}{p} \sum_{n=1}^{\infty} \frac{1}{n\left(n+\frac{2}{p}\right)}+2 p-\frac{1}{p} \sum_{n=1}^{\infty} \frac{1}{n\left(n+\frac{1}{p}\right)}-p \\
& =\frac{1}{p} \sum_{n=1}^{\infty} \frac{1}{\left(n+\frac{1}{p}\right)\left(n+\frac{2}{p}\right)}>0 .
\end{aligned}
$$

Since $h(p) \rightarrow 0$ as $p \rightarrow 0^{+}$and $h^{\prime}(p)>0$ on $(0, \infty)$, it follows that the function $h(p)$ is positive and strictly increasing on $(0, \infty)$.

The reciprocal of $M_{p}(a, b)$ can be expanded into an infinite series. This expansion will be seen to have several uses. Let us introduce the following expressions:

$$
(a, k)=a(a+1) \cdots(a+k-1), \quad(a, 0)=1, \quad(a,-1)=0, \quad a \neq 0 .
$$

Theorem 3 Given $a, b>0$ and $p \in(0, \infty)$, the following expansion holds:

$$
\frac{1}{M_{p}(a, b)}=\frac{1}{\max (a, b)} \sum_{k=0}^{\infty} \frac{\left(\frac{1}{p}, k\right)}{\left(\frac{2}{p}, k\right) k !}\left[1-\left(\frac{\min (a, b)}{\max (a, b)}\right)^{p}\right]^{k}
$$

with $0 !=1$

Proof Both sides of (15) are equal to $1 / a$ when $a=b$. Assume without loss of generality that $a>b>0$. Set $\beta=1-(b / a)^{p}$ so we have $0<\beta<1$ and

$$
a^{p}(1-t)+b^{p} t=a^{p}(1-\beta t) .
$$


Expanding the denominator of (13) into power series gives

$$
\frac{1}{\left[a^{p}(1-t)+b^{p} t\right]^{1 / p}}=\frac{1}{a}(1-\beta t)^{-1 / p}=\frac{1}{a} \sum_{k=0}^{\infty}\left(\frac{1}{p}, k\right) \frac{\beta^{k}}{k !} t^{k} .
$$

The series on the right-hand side of (16) converges when $\beta \in(0,1)$, and so integration of the series term by term is justified. Substitute (16) into (13) to obtain

$$
\begin{aligned}
\frac{1}{M_{p}(a, b)} & =\frac{1}{a B\left(\frac{1}{p}, \frac{1}{p}\right)} \int_{0}^{1} \sum_{k=0}^{\infty} \prod_{m=0}^{k-1}\left(\frac{1}{p}+m\right) \frac{\beta^{k}}{k !} t^{k+1 / p-1}(1-t)^{1 / p-1} d t \\
& =\frac{1}{a B\left(\frac{1}{p}, \frac{1}{p}\right)} \sum_{k=0}^{\infty}\left(\frac{1}{p}, k\right) \frac{\beta^{k}}{k !} \int_{0}^{1} t^{k+1 / p-1}(1-t)^{1 / p-1} d t \\
& =\frac{1}{a} \sum_{k=0}^{\infty}\left(\frac{1}{p}, k\right) \frac{\alpha^{k}}{k !} \frac{B\left(k+\frac{1}{p}, \frac{1}{p}\right)}{B\left(\frac{1}{p}, \frac{1}{p}\right)} .
\end{aligned}
$$

Expressing the beta functions in (17) in terms of the gamma function, we find that

$$
\frac{B\left(k+\frac{1}{p}, \frac{1}{p}\right)}{B\left(\frac{1}{p}, \frac{1}{p}\right)}=\frac{\Gamma\left(k+\frac{1}{p}\right) \Gamma\left(\frac{1}{p}\right)}{\Gamma\left(\frac{1}{p}\right) \Gamma\left(k+\frac{2}{p}\right)}=\prod_{m=0}^{k-1} \frac{\left(\frac{1}{p}+m\right)}{\left(\frac{2}{p}+m\right)}=\frac{\left(\frac{1}{p}, k\right)}{\left(\frac{2}{p}, k\right)} .
$$

Substituting (18) into (17), the required result (15) is obtained.

Another expansion which is relevant to $M_{p}(a, b)$ is given in the following theorem.

Theorem 4 Given $a, b>0$ and $p \in(0, \infty)$,

$$
\frac{1}{M_{p}(a, b)}=\left(\frac{2}{a^{p}+b^{p}}\right)^{1 / p} \sum_{k=0}^{\infty} \frac{\left(\frac{1}{p}, k\right)}{k !} \frac{\left(\frac{1}{p}, 2 k\right)}{\left(\frac{2}{p}, 2 k\right)} \cdot\left(\frac{a^{p}-b^{p}}{a^{p}+b^{p}}\right)^{2 k} .
$$

Proof Completing the square, we can write

$$
\begin{aligned}
\left(x^{p}+a^{p}\right)\left(x^{p}+b^{p}\right) & =\left(x^{p}-\frac{a^{p}+b^{p}}{2}\right)^{2}-\left(\frac{a^{p}-b^{p}}{2}\right)^{2} \\
& =\left(x^{p}+\frac{a^{p}+b^{p}}{2}\right)^{2}(1+\tau(x)),
\end{aligned}
$$

where $\tau(x)$ is defined to be

$$
\tau(x)=\frac{\frac{a^{p}-b^{p}}{2}}{x^{p}+\frac{a^{p}+b^{p}}{2}} .
$$

Clearly, $|\tau(x)|<1$ and using (20), the following expansion holds:

$$
\begin{aligned}
{\left[\left(x^{p}+a^{p}\right)\left(x^{p}+b^{p}\right)\right]^{-1 / p} } & =\left(1+\frac{a^{p}+b^{p}}{2}\right)^{-2 / p}\left(1-\tau(x)^{2}\right)^{-1 / p} \\
& =\left(x^{p}+\frac{a^{p}+b^{p}}{2}\right)^{-2 / p} \sum_{k=0}^{\infty} \frac{\left(\frac{1}{p}, k\right)}{k !} \tau(x)^{k} .
\end{aligned}
$$


Substituting expansion (21) into the integral (13) for $M_{p}(a, b)^{-1}$, we obtain

$$
\begin{aligned}
\frac{1}{M_{p}(a, b)} & =c_{p} \int_{0}^{\infty} \frac{1}{\left(x^{p}+\frac{a^{p}+b^{p}}{2}\right)^{2 / p}} \sum_{k=0}^{\infty} \frac{\left(\frac{1}{p}, k\right)}{k !} \tau(x)^{2 k} d x \\
& =c_{p} \int_{0}^{\infty} \frac{d x}{\left(x^{p}+\frac{a^{p}+b^{p}}{2}\right)^{2 / p}}+c_{p} \sum_{k=1}^{\infty} \frac{\left(\frac{1}{p}, k\right)}{k !} \int_{0}^{\infty} \frac{\tau(x)^{2 k}}{\left(x^{p}+\frac{a^{p}+b^{p}}{2}\right)^{2 / p}} d x \\
& =\left(\frac{a^{p}+b^{p}}{2}\right)^{-1 / p}+c_{p} \sum_{k=1}^{\infty} \frac{\left(\frac{1}{p}, k\right)}{k !} \int_{0}^{\infty} \frac{\tau(x)^{2 k}}{\left(x^{p}+\frac{a^{p}+b^{p}}{2}\right)^{2 / p}} d x \\
& =\left(\frac{2}{a^{p}+b^{p}}\right)^{1 / p}+c_{p} \sum_{k=1}^{\infty} \frac{\left(\frac{1}{p}, k\right)}{k !}\left(\frac{a^{p}-b^{p}}{2}\right)^{2 k} \int_{0}^{\infty} \frac{d x}{\left(x^{p}+\frac{a^{p}+b^{p}}{2}\right)^{2 k+2 / p}} .
\end{aligned}
$$

Consider the integral apart from (22) and make use of the substitution

$$
x=\left(\frac{a^{p}+b^{p}}{2}\right)^{1 / p} y
$$

The integral in (22) takes the form

$$
\int_{0}^{\infty} \frac{d x}{\left(x^{p}+\frac{a^{p}+b^{p}}{2}\right)^{2 k+2 / p}}=\left(\frac{a^{p}+b^{p}}{2}\right)^{-2 k-1 / p} \int_{0}^{\infty} \frac{d y}{\left(y^{p}+1\right)^{2 k+2 / p}}
$$

Finally, introduce the change of variable $y^{p}+1=t^{-1}$ into the integral so it takes the form

$$
\int_{0}^{\infty} \frac{d x}{\left(x^{p}+\frac{a^{p}+b^{p}}{2}\right)^{2 k+2 / p}}=\left(\frac{a^{p}+b^{p}}{2}\right)^{-2 k-1 / p} \frac{1}{p} B\left(2 k+\frac{1}{p}, \frac{1}{p}\right) .
$$

Multiply (23) by $c_{p}$ from (12) to obtain

$$
c_{p} \int_{0}^{\infty} \frac{d x}{\left(x^{p}+\frac{a^{p}+b^{p}}{2}\right)^{2 k+2 / p}}=\left(\frac{a^{p}+b^{p}}{2}\right)^{-2 k-1 / p} \frac{B\left(2 k+\frac{1}{p}, \frac{1}{p}\right)}{B\left(\frac{1}{p}, \frac{1}{p}\right)}=\left(\frac{2}{a^{p}+b^{p}}\right)^{2 k+1 / p\left(\frac{1}{p}, 2 k\right)} \frac{\left.\frac{2}{p}, 2 k\right)}{.}
$$

Substituting this integral into (22), we arrive at the desired expansion

$$
\frac{1}{M_{p}(a, b)}=\left(\frac{2}{a^{p}+b^{p}}\right)^{1 / p}+\left(\frac{2}{a^{p}+b^{p}}\right)^{1 / p} \sum_{k=1}^{\infty} \frac{\left(\frac{1}{p}, k\right)}{k !} \frac{\left(\frac{1}{p}, 2 k\right)}{\left(\frac{2}{p}, 2 k\right)}\left(\frac{a^{p}-b^{p}}{a^{p}+b^{p}}\right)^{2 k} .
$$

\section{Bounds for $M_{p}(a, b)$}

Theorem 5 Given $a, b>0$, the following set of inequalities holds:

$$
M_{0}(a, b)=\sqrt{a b} \leq M_{p}(a, b), \quad M_{\infty}(a, b)=\frac{2 \max (a, b)}{2+\log \left(\frac{\max (a, b)}{\min (a, b)}\right)} \leq \frac{a+b}{2}
$$

Proof It can be verified that

$$
\lim _{p \rightarrow 0^{+}}\left(\frac{a^{p}+b^{p}}{2}\right)^{1 / p}=\sqrt{a b}
$$


so the equality $M_{0}(a, b)=\sqrt{a b}$ follows directly from Theorem 1 . The inequalities on either side of $M_{p}(a, b)$ follow from monotonicity of $M_{p}(a, b)$ as a function of $p$. The last two inequalities follow easily if $a=b$. Suppose then that $a>b>0$. Since it has been shown that $c_{p} \rightarrow 1 / 2$ as $p \rightarrow \infty$, we conclude that

$$
\lim _{p \rightarrow \infty} \int_{0}^{\infty} \frac{d y}{\left(y^{p}+1\right)^{2 / p}}=2
$$

Moreover,

$$
\begin{aligned}
\lim _{p \rightarrow \infty} \int_{0}^{\infty} \frac{d x}{\left[\left(x^{p}+a^{p}\right)\left(x^{p}+b^{p}\right)\right]^{1 / p}} & =\int_{0}^{b} \frac{d x}{a b}+\int_{b}^{a} \frac{d x}{x a}+\int_{a}^{\infty} \frac{d x}{x^{2}} \\
& =\frac{2+(\log (a)-\log (b))}{a}=\frac{2+\log \left(\frac{a}{b}\right)}{a} .
\end{aligned}
$$

Therefore,

$$
\lim _{p \rightarrow \infty} M_{p}(a, b)=\frac{2 a}{2+\log \left(\frac{a}{b}\right)}
$$

Finally, it is the case that the mean $M_{1}(a, b)$ satisfies the inequality

$$
\frac{a-b}{\log (a)-\log (b)} \leq \frac{a+b}{2}
$$

Consequently, this implies the following inequality:

$$
2(a-b) \leq(a+b)(\log (a)-\log (b)) .
$$

Collecting terms on the right and adding $2 a$ to both sides, we get

$$
4 a \leq 2(a+b)+(a+b)(\log (a)-\log (b)) .
$$

This result implies the upper bound for $M_{\infty}(a, b)$,

$$
\frac{2 a}{2+\log \left(\frac{a}{b}\right)} \leq \frac{a+b}{2} .
$$

This is the final inequality on the right in (24), so we are done.

Lemma 1 For fixed $p>0$ and $k \in \mathbb{N}$,

$$
\frac{\left(\frac{1}{p}+k\right)^{2}}{k\left(\frac{2}{p}+k\right)} \geq 1 .
$$

Proof Since $(1 / p)^{2} \geq 0$, it follows that

$$
\left(\frac{1}{p}\right)^{2}+2 \frac{k}{p}+k^{2} \geq 2 \frac{k}{p}+k^{2}
$$


Consequently,

$$
\left(\frac{1}{p}+k\right)^{2} \geq k\left(\frac{2}{p}+k\right)
$$

Dividing both sides of this by the right-hand side, (25) is obtained.

Theorem 6 For $a, b>0$ and all $p \in(0, \infty)$, the following bound holds:

$$
M_{p}(a, b) \leq M_{\infty}(a, b)
$$

Proof The proof relies on expressing series (15) given in Theorem 3 and (24) in a certain way. In fact, the reciprocal of (26) will be shown. Let $p>0$ and define

$$
r=r_{p}=1-\left(\frac{\min (a, b)}{\max (a, b)}\right)^{p} .
$$

Clearly, $r \in(0,1)$ and this can be solved for the ratio on the right as a function of $r$,

$$
\frac{\max (a, b)}{\min (a, b)}=(1-r)^{-1 / p} .
$$

From Theorem 3, the following expansion holds for any $p \in(0, \infty)$ :

$$
\frac{\max (a, b)}{M_{p}(a, b)}=1+\sum_{k=1}^{\infty} \frac{\left(\frac{1}{p}, k\right)^{2}}{\left(\frac{2}{p}, k\right) k !} r^{k} .
$$

Moreover, substituting (27) into (24), with $r \in(0,1)$, expand the logarithm function in series to obtain

$$
\frac{\max (a, b)}{M_{\infty}(a, b)}=1+\frac{1}{2 p} \sum_{k=1}^{\infty} \frac{r^{k}}{k} .
$$

Therefore, it suffices to show the reciprocal of (26) holds,

$$
\sum_{k=1}^{\infty} \frac{\left(\frac{1}{p}, k\right)^{2}}{\left(\frac{2}{p}, k\right) k !} r^{k} \geq \frac{1}{2 p} \sum_{k=1}^{\infty} \frac{r^{k}}{k} .
$$

This is equivalent to the inequality

$$
\sum_{k=1}^{\infty}\left[\frac{\left(\frac{1}{p}, k\right)^{2}}{\left(\frac{2}{q}, k\right) k !}-\frac{1}{2 p k}\right] r^{k} \geq 0 .
$$

If it can be shown that the coefficients in (30) are positive for each $k$, inequality in (30) will follow. This amounts to showing that

$$
\frac{\left(\frac{1}{p}, k\right)^{2}}{\left(\frac{2}{p}, k\right) k !} \geq \frac{1}{2 p k} .
$$


This implies it has to be shown that

$$
2 p\left(\frac{1}{p}, k\right)^{2} \geq\left(\frac{2}{p}, k\right)(k-1) !
$$

It is clear that when $k=1$ and 2 are put in (31) the inequality holds. Suppose (31) holds up to some value of $k$, so the following statement holds

$$
2 p\left(\frac{1}{p}\right)^{2}\left(\frac{1}{p}+1\right)^{2} \cdots\left(\frac{1}{p}+k-1\right)^{2} \geq \frac{2}{p}\left(\frac{2}{p}+1\right) \cdots\left(\frac{2}{p}+k-1\right)(k-1) ! .
$$

Multiply both sides of (32) by $\left(\frac{1}{p}+k\right)^{2}$ and use (25) from Lemma 1 to obtain that

$$
\begin{aligned}
2 p & \left(\frac{1}{p}\right)^{2} \cdots\left(\frac{1}{p}+k-1\right)^{2}\left(\frac{1}{p}+k\right)^{2} \\
& \geq \frac{2}{p}\left(\frac{2}{p}+1\right) \cdots\left(\frac{2}{p}+k-1\right)\left(\frac{2}{p}+k\right) k ! \cdot\left(\frac{\left(\frac{1}{p}+k\right)^{2}}{k\left(\frac{2}{p}+k\right)}\right) \\
& \geq \frac{2}{p}\left(\frac{2}{p}+1\right) \cdots\left(\frac{2}{p}+k\right) k ! .
\end{aligned}
$$

This is exactly (32) but with $k$ replaced by $k+1$. By the Principle of Mathematical Induction, (32) holds.

This serves to generalize the result given in [4] where it was shown that $M_{2}(a, b) \leq$ $M_{\infty}(a, b)$.

\section{Acknowledgements}

I gratefully acknowledge the publication fee waiver from Springer.

Funding

No funding source, but I gratefully acknowledge a publication fee waiver from Springer.

Availability of data and materials

No other data or material was involved.

Competing interests

No competing interests. Paper was completed as part of research component of my work.

Authors' contributions

Paper was entirely the work of the sole author P Bracken. All authors read and approved the final manuscript.

\section{Authors' information}

The author is a full Professor at University of Texas, Edinburg, TX.

\section{Publisher's Note}

Springer Nature remains neutral with regard to jurisdictional claims in published maps and institutional affiliations.

Received: 4 February 2019 Accepted: 1 July 2019 Published online: 17 July 2019

\section{References}

1. Gauss, C.F.: Werke, vol. 3, Göttingen, 1866-1933

2. Borwein, J.M., Borwein, P.B.: Pi and the AGM. Wiley, New York (1987)

3. Bracken, P.: An arithmetic-geometric mean inequality. Expo. Math. 19, 273-279 (2001)

4. Batia, R., Li, R.-C.: An Interpolating Family of Means. UT Arlington Preprint Series, 2011-02

5. Carlson, B.C., Vuorinen, M.: Problem 91-17. SIAM Rev. 34, 653 (1992)

6. Huang, T.-R., Tan, S.-T., Ma, X.-Y., Chu, Y.-M.: Monotonicity properties and bounds for the complete $p$-elliptic integrals. J. Inequal. Appl. 2018, 239 (2018)

7. Nakamura, N.: Order relations among some interpolating families of means. Toyama Math. J. 35, 35-48 (2012) 